\title{
On the spectra of general random mixed graphs
}

\author{
Dan $\mathrm{Hu}^{*}$ \\ School of Sciences \\ Xi'an University of Technology \\ Xi'an, Shaanxi 710048, P.R. China \\ danhu@xaut.edu.cn \\ Jiangyou Hou $\ddagger$ \\ School of Mathematics \\ Northwest University \\ Xi'an, Shaanxi 710127, P.R. China \\ jiangyonghou@nwu.edu.cn
}

\author{
Hajo Broersma ${ }^{\dagger}$ \\ Faculty of EEMCS \\ University of Twente \\ P.O. Box 217, 7500 AE Enschede, The Netherlands \\ h.j.broersma@utwente.nl
}

\author{
Shenggui Zhang $\S$ \\ School of Mathematics and Statistics, and \\ Xi'an-Budapest Joint Research Center \\ for Combinatorics \\ Northwestern Polytechnical University \\ Xi'an, Shaanxi 710129, P.R. China \\ shengguizhang@nwpu .edu.au
}

Submitted: Jun 10, 2020; Accepted: Dec 7, 2020; Published: Jan 15, 2021

(C) The authors. Released under the CC BY-ND license (International 4.0).

\begin{abstract}
A mixed graph is a graph that can be obtained from a simple undirected graph by replacing some of the edges by arcs in precisely one of the two possible directions. The Hermitian adjacency matrix of a mixed graph $G$ of order $n$ is the $n \times n$ matrix $H(G)=\left(h_{i j}\right)$, where $h_{i j}=-h_{j i}=\mathbf{i}$ (with $\mathbf{i}=\sqrt{-1}$ ) if there exists an arc from $v_{i}$ to $v_{j}$ (but no arc from $v_{j}$ to $v_{i}$ ), $h_{i j}=h_{j i}=1$ if there exists an edge (and no arcs) between $v_{i}$ and $v_{j}$, and $h_{i j}=0$ otherwise (if $v_{i}$ and $v_{j}$ are neither joined by an edge nor by an arc). We study the spectra of the Hermitian adjacency matrix and the normalized Hermitian Laplacian matrix of general random mixed graphs, i.e., in which all arcs are chosen independently with different probabilities (and an edge is regarded as two oppositely oriented arcs joining the same pair of vertices). For our first main result, we derive a new probability inequality and apply it to obtain an upper bound on the eigenvalues of the Hermitian adjacency matrix. Our
\end{abstract}

\footnotetext{
*Supported by NSFC (No. 12001421), Scientific Research Program Funded by Shaanxi Provincial Education Department (20JK0782).

$\dagger$ Corresponding author.

¥Supported by NSFC (No. 11701451).

$\S$ Supported by NSFC (No. 12071370 and U1803263).
} 
second main result shows that the eigenvalues of the normalized Hermitian Laplacian matrix can be approximated by the eigenvalues of a closely related weighted expectation matrix, with error bounds depending on the minimum expected degree of the underlying undirected graph.

Keywords: general random mixed graphs; random Hermitian adjacency matrix; random normalized Hermitian Laplacian matrix; spectra

Mathematics Subject Classifications: 05C50, 15A18

\section{Introduction}

A Hermitian matrix (also sometimes called self-adjoint matrix) is a complex square matrix that is equal to its own conjugate transpose, i.e., the $(i, j)$-th element is equal to the complex conjugate of the $(j, i)$-th element, for all indices $i$ and $j$. The purpose of this paper is to study the spectra of the Hermitian adjacency matrix and the normalized Hermitian Laplacian matrix of general random mixed graphs. Before we give the other necessary definitions and present our results, we start off with a short overview and some background on related work.

\subsection{Background and related work}

Spectra of the adjacency matrix and the normalized Laplacian matrix of graphs have many applications in graph theory. For example, the spectrum of the adjacency matrix of a graph is related to its connectivity and the number of occurrences of specific subgraphs, and also to its chromatic number and its independence number. The spectrum of the normalized Laplacian matrix is related to diffusion on graphs, random walks on graphs and the Cheeger constant. For more details on these notions, and for more applications of spectra of the adjacency matrix and the normalized Laplacian matrix, we refer the interested reader to two monographs [5, 12].

Also for random graphs, spectra of their adjacency matrix and their normalized Laplacian matrix are well-studied (See, e.g., $[2,6,7,9,10,13,14,17,18]$ ). We next present a brief account of some of the results that were obtained for random graphs. We refrain from giving an exhaustive overview, and we refer the reader to the sources for more background, and for terminology and notation.

Given a graph $G$ of order $n$, let $\mu_{1}, \ldots, \mu_{n}$ be the eigenvalues of the adjacency matrix of $G$ in nonincreasing order. Adopting the Erdős-Rényi random graph model $G(n, p)$, Füredi and Komlós [18] showed that asymptotically almost surely $\mu_{1}=(1+o(1)) n p$ and $\max \left\{\mu_{2},-\mu_{n-1}\right\} \leqslant(2+o(1)) \sqrt{n p(1-p)}$ provided $n p(1-p) \gg \log ^{6} n$. Here the notation $o(1)$ is used for a quantity (depending on $n$ ) that goes to 0 as $n$ goes to infinity. These results were extended to sparse random graphs $[14,22]$ and general random matrices $[13,18]$. Tropp [29] determined probability inequalities for sums of independent random self-adjoint matrices. Alon, Krivelevich, and $\mathrm{Vu}[2]$ studied the concentration of the $s$-th largest eigenvalue of a random symmetric matrix with independent random entries of absolute value at most one. Friedman et al. $[15,16,17]$ proved that the second largest 
eigenvalue (in absolute value) of random $d$-regular graphs is almost surely $(2+o(1)) \sqrt{d-1}$ for any $d \geqslant 4$. Chung, $\mathrm{Lu}$, and $\mathrm{Vu}[7]$ studied spectra of the adjacency matrix of random power law graphs, and spectra of the normalized Laplacian matrix of random graphs with given expected degrees. Their results on random graphs with given expected degree sequences were supplemented by Coja-Oghlan et al. $[9,10]$ for sparse random graphs. Lu and Peng $[24,25]$ studied spectra of the adjacency matrix and the normalized Laplacian matrix of edge-independent random graphs, as well as spectra of the normalized Laplacian matrix of random hypergraphs. Oliveira [26] considered the problem of approximating the spectra of the adjacency matrix and the normalized Laplacian matrix of random graphs. His results were improved by Chung and Radcliffe [8].

The purpose of our paper is to extend these studies to general random mixed graphs.

\subsection{General random mixed graphs}

A graph is called a mixed graph if it contains both directed and undirected edges. We use $G=(V(G), E(G), A(G))$ to denote a mixed graph with a set $V(G)$ of vertices, a set $E(G)$ of (undirected) edges, and a set $A(G)$ of arcs (directed edges). We define the underlying graph of $G$, denoted by $\Gamma(G)$, as the graph with vertex set $V(\Gamma(G))=V(G)$, and edge set

$$
E(\Gamma(G))=\left\{v_{i} v_{j} \mid v_{i} v_{j} \in E(G) \text { or }\left(v_{i}, v_{j}\right) \in A(G) \text { or }\left(v_{j}, v_{i}\right) \in A(G)\right\} .
$$

We adopt the terminology and notation of Liu and $\mathrm{Li}$ in [23], and define the Hermitian adjacency matrix of a mixed graph $G$ of order $n$ to be the $n \times n$ matrix $H(G)=\left(h_{i j}\right)_{n \times n}$, where

$$
h_{i j}=\left\{\begin{aligned}
1, & \text { if } v_{i} v_{j} \in E(G) ; \\
\mathbf{i}, & \text { if }\left(v_{i}, v_{j}\right) \in A(G) \text { and }\left(v_{j}, v_{i}\right) \notin A(G) ; \\
-\mathbf{i}, & \text { if }\left(v_{i}, v_{j}\right) \notin A(G) \text { and }\left(v_{j}, v_{i}\right) \in A(G) ; \\
0, & \text { otherwise. }
\end{aligned}\right.
$$

Here, $\mathbf{i}=\sqrt{-1}$. This matrix, that is indeed Hermitian, as one easily sees, was also introduced independently by Guo and Mohar in [20]. We denote by $\lambda_{i}(H(G))$ the $i$-th largest eigenvalue of $H(G)$ (multiplicities counted). We use $\left\{\lambda_{1}(H(G)), \lambda_{2}(H(G)), \ldots, \lambda_{n}(H(G))\right\}$ to denote the spectrum of $H(G)$ in nonincreasing order. The set of these eigenvalues is called the Hermitian adjacency spectrum (or briefly $H$-spectrum) of $G$. Let $V(G)=$ $\left\{v_{1}, v_{2}, \ldots, v_{n}\right\}$, and let $D(G)=\operatorname{diag}\left(d_{1}, d_{2}, \ldots, d_{n}\right)$ be a diagonal matrix, in which $d_{i}$ is the degree of the vertex $v_{i}$ in the underlying graph $\Gamma(G)$. Then the matrix $L(G)=$ $D(G)-H(G)$ is called the Hermitian Laplacian matrix of $G$, and the matrix $\mathcal{L}(G)=$ $I-D(G)^{-\frac{1}{2}} H(G) D(G)^{-\frac{1}{2}}$ is called the normalized Hermitian Laplacian matrix of $G$. Here $I$ is the $n \times n$ identity matrix. We denote by $\lambda_{i}(\mathcal{L}(G))$ the $i$-th largest eigenvalue of $\mathcal{L}(G)$ (multiplicities counted). We use $\left\{\lambda_{1}(\mathcal{L}(G)), \lambda_{2}(\mathcal{L}(G)), \ldots, \lambda_{n}(\mathcal{L}(G))\right\}$ to denote the spectrum of $\mathcal{L}(G)$ in nonincreasing order. The set of these eigenvalues is called the normalized Hermitian Laplacian spectrum of $G$.

If we regard (replace) each edge $v_{i} v_{j} \in E(G)$ in $G=(V(G), E(G), A(G))$ as (by) two oppositely directed $\operatorname{arcs}\left(v_{i}, v_{j}\right)$ and $\left(v_{j}, v_{i}\right)$, then $G$ is a directed graph. Throughout this paper, we regard mixed graphs as directed graphs, in the above sense. Next, we give the 
definition of a general random mixed graph $\widehat{G}_{n}\left(p_{i j}\right)$. Let $K_{n}$ be a complete graph on $n$ vertices. The complete directed graph $D K_{n}$ is the graph obtained from $K_{n}$ by replacing each edge of $K_{n}$ by two oppositely directed arcs. Let $p_{i j}$ be a function of $n$ such that $0<p_{i j}<1(i \neq j)$. We always assume that $p_{i i}=0$ for all indices $i$. The random mixed graph model $\widehat{\mathcal{G}}_{n}\left(p_{i j}\right)$ consists of all random mixed graphs $\widehat{G}_{n}\left(p_{i j}\right)$ in which each arc $\left(v_{i}, v_{j}\right)$ with $i \neq j$ is chosen randomly and independently, with probability $p_{i j}$ from the set of arcs of $D K_{n}$, where we let the vertex set be $\left\{v_{1}, v_{2}, \ldots, v_{n}\right\}$. Here the probabilities $p_{i j}$ for different arcs are not assumed to be equal, that is, $\widehat{G}_{n}\left(p_{i j}\right)$ is an arc-independent random mixed graph of order $n$. Then the Hermitian adjacency matrix of $\widehat{G}_{n}\left(p_{i j}\right)$, denoted by $H\left(\widehat{G}_{n}\left(p_{i j}\right)\right)=\left(h_{i j}\right)$ (or $H_{n}$, for brevity), satisfies that:

- $H_{n}$ is a random Hermitian matrix, with $h_{i i}=0$ for $1 \leqslant i \leqslant n$;

- the upper-triangular entries $h_{i j}, 1 \leqslant i<j \leqslant n$ are independent random variables, which take value 1 with probability $p_{i j} p_{j i}$, i with probability $p_{i j}\left(1-p_{j i}\right),-\mathbf{i}$ with probability $\left(1-p_{i j}\right) p_{j i}$, and 0 with probability $\left(1-p_{i j}\right)\left(1-p_{j i}\right)$.

If $A$ is a random $n \times n$ matrix, we write $\mathbb{E}(A)$ to denote the coordinate-wise expectation of $A$, so $\mathbb{E}(A)_{i j}=\mathbb{E}\left(A_{i j}\right)$. Similarly, $\operatorname{Var}(A)=\mathbb{E}\left((A-\mathbb{E}(A))^{2}\right)$. We use $\mathbb{E} H_{n}$ as shorthand for $\mathbb{E}\left(H_{n}\right)$, and note that it is obvious that $\left(\mathbb{E} H_{n}\right)_{i j}=\mathbb{E}\left(h_{i j}\right)=p_{i j} p_{j i}+\mathbf{i}\left(p_{i j}-p_{j i}\right)$.

\subsection{Our main results}

In this paper we study the spectra of the Hermitian adjacency matrix and the normalized Hermitian Laplacian matrix of general random mixed graphs. Throughout adopting the above notation and terminology, our main results can be stated as follows.

For the first theorem, we use $\Delta\left(\Gamma\left(\widehat{G}_{n}\left(p_{i j}\right)\right)\right)$ to denote the maximum expected degree of the underlying graph of $\widehat{G}_{n}\left(p_{i j}\right)$. Hence, by straightforward calculations, $\Delta\left(\Gamma\left(\widehat{G}_{n}\left(p_{i j}\right)\right)\right)=$ $\max _{1 \leqslant i \leqslant n} \sum_{j=1}^{n}\left(p_{i j}+p_{j i}-p_{i j} p_{j i}\right)$.

Theorem 1. Let $\widehat{G}_{n}\left(p_{i j}\right)$ and $H_{n}=\left(h_{i j}\right)$ be defined as above, and let $\Delta=\Delta\left(\Gamma\left(\widehat{G}_{n}\left(p_{i j}\right)\right)\right)$. Let $\epsilon>0$ be an arbitrarily small constant, chosen such that for $n$ sufficiently large, $\Delta>\frac{4}{9} \ln (2 n / \epsilon)$. Then with probability at least $1-\epsilon$, for $n$ sufficiently large, the eigenvalues of $H_{n}$ satisfy

$$
\left|\lambda_{i}\left(H_{n}\right)\right| \leqslant \max _{1 \leqslant i \leqslant n} \sum_{j=1}^{n} \sqrt{p_{i j}^{2} p_{j i}^{2}+\left(p_{i j}-p_{j i}\right)^{2}}+2 \sqrt{\Delta \ln (2 n / \epsilon)}
$$

for all $1 \leqslant i \leqslant n$.

Applying Theorem 1 with $\epsilon=\frac{1}{n}$, we immediately obtain the following corollaries.

Corollary 2. Let $\widehat{G}_{n}\left(p_{i j}\right)$ and $H_{n}=\left(h_{i j}\right)$ be defined as in Theorem 1, and let $\Delta=$ $\Delta\left(\Gamma\left(\widehat{G}_{n}\left(p_{i j}\right)\right)\right)$. If $\Delta>\frac{8}{9} \ln (\sqrt{2} n)$, then with probability at least $1-\frac{1}{n}=1-o(1)$, the 
eigenvalues of $H_{n}$ satisfy

$$
\left|\lambda_{i}\left(H_{n}\right)\right| \leqslant \max _{1 \leqslant i \leqslant n} \sum_{j=1}^{n} \sqrt{p_{i j}^{2} p_{j i}^{2}+\left(p_{i j}-p_{j i}\right)^{2}}+2 \sqrt{2 \Delta \ln (\sqrt{2} n)}
$$

for all $1 \leqslant i \leqslant n$.

In particular, consider the case that $p_{i j}=p$, with $p \in(0,1)$ for $i \neq j$ and 0 for $i=j$. We denote this graph by $\widehat{G}_{n}(p)$ and define

$$
\begin{aligned}
\beta & :=\frac{2 \sqrt{2 \Delta \ln (\sqrt{2} n)}}{\max _{1 \leqslant i \leqslant n} \sum_{j=1}^{n} \sqrt{p_{i j}^{2} p_{j i}^{2}+\left(p_{i j}-p_{j i}\right)^{2}}} \\
& =\frac{2 \sqrt{2\left(2 p-p^{2}\right)(n-1) \ln (\sqrt{2} n)}}{p^{2}(n-1)} \\
& =2 \sqrt{2} \cdot \sqrt{\frac{2-p}{p^{3}} \cdot \frac{\ln (\sqrt{2} n)}{n-1}} .
\end{aligned}
$$

- If $p$ is a constant, then $\beta \rightarrow 0$ as $n \rightarrow \infty$.

- If $p \rightarrow 1$, then $\beta \rightarrow 0$ as $n \rightarrow \infty$.

- Let $p=\frac{1}{n^{l}}$. If $0<l<\frac{1}{3}$, then $\beta \rightarrow 0$ as $n \rightarrow \infty$; if $l \geqslant \frac{1}{3}$, then $\beta \rightarrow \infty$ as $n \rightarrow \infty$.

Corollary 3. For $\widehat{G}_{n}(p)$, if $(n-1)\left(2 p-p^{2}\right)>\frac{8}{9} \ln (\sqrt{2} n)$, then with probability at least $1-\frac{1}{n}=1-o(1)$, we have

$$
\left|\lambda_{i}\left(H_{n}\right)\right| \leqslant(1+o(1))(n-1) p^{2}, \text { for all } 1 \leqslant i \leqslant n,
$$

when $p$ is a constant or $p \rightarrow 1$ or $p=\frac{1}{n^{l}}, 0<l<\frac{1}{3}$.

In the next theorem, we assume that $V\left(\widehat{G}_{n}\left(p_{i j}\right)\right)=\left\{v_{1}, v_{2}, \ldots, v_{n}\right\}$, and we let $D_{n}=$ $\operatorname{diag}\left(d_{1}, d_{2}, \ldots, d_{n}\right)$ denote the diagonal matrix in which $d_{i}$ is the degree of the vertex $v_{i}$ in the underlying graph of $\widehat{G}_{n}\left(p_{i j}\right)$. We let $\mathbb{E} D_{n}$ denote the coordinate-wise expectation of $D_{n}$. Recall that $\mathcal{L}_{n}=I_{n}-D_{n}^{-1 / 2} H_{n} D_{n}^{-1 / 2}$ denotes the normalized Hermitian Laplacian matrix of $\widehat{G}_{n}\left(p_{i j}\right)$, where $I_{n}$ denotes the $n \times n$ identity matrix. We let $\delta\left(\Gamma\left(\widehat{G}_{n}\left(p_{i j}\right)\right)\right)$ denote the minimum expected degree of the underlying graph of $\widehat{G}_{n}\left(p_{i j}\right)$. Hence, $\delta\left(\Gamma\left(\widehat{G}_{n}\left(p_{i j}\right)\right)\right)=$ $\min _{1 \leqslant i \leqslant n} \sum_{j=1}^{n}\left(p_{i j}+p_{j i}-p_{i j} p_{j i}\right)$.

Theorem 4. Let $\widehat{G}_{n}\left(p_{i j}\right), H_{n}, D_{n}$ and $\mathcal{L}_{n}$ be defined as above, and let $\delta=\delta\left(\Gamma\left(\widehat{G}_{n}\left(p_{i j}\right)\right)\right)$. Let $\epsilon>0$ be an arbitrarily small constant. Then there exists a constant $k=k(\epsilon)$ such that if $\delta>k \ln n$, then with probability at least $1-\epsilon$, the eigenvalues of $\mathcal{L}_{n}$ and $\widetilde{\mathcal{L}_{n}}$ satisfy

$$
\left|\lambda_{i}\left(\mathcal{L}_{n}\right)-\lambda_{i}\left(\widetilde{\mathcal{L}_{n}}\right)\right| \leqslant 7 \sqrt{\frac{\ln (4 n / \epsilon)}{\delta}}
$$

for all $1 \leqslant i \leqslant n$, where $\widetilde{\mathcal{L}_{n}}=I_{n}-\left(\mathbb{E} D_{n}\right)^{-1 / 2}\left(\mathbb{E} H_{n}\right)\left(\mathbb{E} D_{n}\right)^{-1 / 2}$. 
Applying Theorem 4 with $\epsilon=\frac{1}{n}$, we immediately obtain the following corollaries.

Corollary 5. Let $\widehat{G}_{n}\left(p_{i j}\right), H_{n}, D_{n}$ and $\mathcal{L}_{n}$ be defined as in Theorem 4 , and let $\delta=$ $\delta\left(\Gamma\left(\widehat{G}_{n}\left(p_{i j}\right)\right)\right)$. If $\delta \gg \ln n$, then with probability at least $1-\frac{1}{n}=1-o(1)$, the eigenvalues of $\mathcal{L}_{n}$ and $\widetilde{\mathcal{L}_{n}}$ satisfy

$$
\left|\lambda_{i}\left(\mathcal{L}_{n}\right)-\lambda_{i}\left(\widetilde{\mathcal{L}_{n}}\right)\right| \leqslant 7 \sqrt{\frac{2 \ln (2 n)}{\delta}}=o(1)
$$

for all $1 \leqslant i \leqslant n$, where $\widetilde{\mathcal{L}_{n}}=I_{n}-\left(\mathbb{E} D_{n}\right)^{-1 / 2}\left(\mathbb{E} H_{n}\right)\left(\mathbb{E} D_{n}\right)^{-1 / 2}$.

If $J_{n}$ is the $n \times n$ all-ones matrix, then for $\widehat{G}_{n}(p), \mathbb{E} H_{n}=p^{2}\left(J_{n}-I_{n}\right), \mathbb{E} D_{n}=(n-$ 1) $\left(2 p-p^{2}\right) I_{n}$ and $\widetilde{\mathcal{L}_{n}}=I_{n}-\frac{p}{(n-1)(2-p)}\left(J_{n}-I_{n}\right)$. Then we have the following.

Corollary 6. For $\widehat{G}_{n}(p)$, if $(n-1)\left(2 p-p^{2}\right) \gg \ln n$, then with probability at least $1-\frac{1}{n}=$ $1-o(1)$, we have

$$
\left|\lambda_{i}\left(\mathcal{L}_{n}\right)-\lambda_{i}\left(I_{n}-\frac{p}{(n-1)(2-p)}\left(J_{n}-I_{n}\right)\right)\right| \leqslant 7 \sqrt{\frac{2 \ln (2 n)}{(n-1)\left(2 p-p^{2}\right)}}=o(1)
$$

for all $1 \leqslant i \leqslant n$.

The spectrum of $I_{n}-\frac{p}{(n-1)(2-p)}\left(J_{n}-I_{n}\right)$ is $\left\{1-\frac{p}{2-p}, 1+\frac{p}{(n-1)(2-p)}\right\}$, where $1+\frac{p}{(n-1)(2-p)}$ has multiplicity $n-1$. From Corollary 6 , we see that, if $(n-1)\left(2 p-p^{2}\right) \gg \ln n$ and $p \rightarrow 0$, then with high probability all eigenvalues of $\mathcal{L}_{n}$ are close to 1 ; otherwise, if $(n-1)\left(2 p-p^{2}\right) \gg \ln n$ and $p \nrightarrow 0$, all eigenvalues of $\mathcal{L}_{n}$ except $\lambda_{\min }\left(\mathcal{L}_{n}\right)$ are close to 1 .

We postpone the proofs of Theorem 1 and Theorem 4 to Section 3. In the next section, we state some existing results and prove an auxiliary new result that we need in our proof of Theorem 1.

\section{Preliminaries and Auxiliary Results}

We start with some additional terminology and notation that we will use throughout the paper.

\subsection{Additional terminology and notation}

We let $\mathbb{C}_{\text {Herm }}^{n \times n}$ denote the set of $n \times n$ Hermitian matrices, which is a subset of the set $\mathbb{C}^{n \times n}$ of all $n \times n$ matrices with complex entries. For each matrix $M \in \mathbb{C}^{n \times n}$, the spectral radius of $M$ is the nonnegative real number $\rho(M)=\max \left\{\left|\lambda_{i}(M)\right|: 1 \leqslant i \leqslant n\right\}$, where $\lambda_{i}(M)$ $(1 \leqslant i \leqslant n)$ are all eigenvalues of $M$. We use $\lambda_{\max }(M)$ to denote the largest eigenvalue of $M$. The set $\left\{\lambda_{i}(M): 1 \leqslant i \leqslant n\right\}$ is called the spectrum of $M$, and denoted by $\operatorname{spec}(M)$. The spectral norm $\|M\|$ is the largest singular value of $M$, i.e., we have

$$
\|M\|=\sqrt{\lambda_{\max }\left(M^{*} M\right)} .
$$


Here $M^{*}$ is the conjugate transpose of $M$. The Spectral Theorem for Hermitian matrices states that all $M \in \mathbb{C}_{H e r m}^{n \times n}$ have $n$ real eigenvalues (possibly with repetitions) that correspond to an orthonormal set of eigenvectors.

When $M \in \mathbb{C}_{\text {Herm }}^{n \times n}$, we have $\|M\|=\max \left\{\left|\lambda_{i}(M)\right|: 1 \leqslant i \leqslant n\right\}$. Then $\rho(M)=\|M\|=$ $\max \left\{\lambda_{\max }(M), \lambda_{\max }(-M)\right\}$. We use $\operatorname{Tr}(M)$ (the trace of $M$ ) to denote the sum of the eigenvalues of $M$.

We will use the notation $A \succeq \mathbf{0}$ to indicate that $A$ is positive semidefinite, i.e., $A \in$ $\mathbb{C}_{\text {Herm }}^{n \times n}$ and its eigenvalues are nonnegative, and use the notation $A \succ \mathbf{0}$ to indicate that $A$ is positive definite, i.e., $A \in \mathbb{C}_{\text {Herm }}^{n \times n}$ and its eigenvalues are positive, where $\mathbf{0}$ is the zero matrix of the same size as $A$. With $\preceq$ we denote the positive semidefinite order on Hermitian matrices, as follows. Given two Hermitian matrices $A$ and $B$, we use $A \preceq B$ or $B \succeq A$ to indicate that $B-A \succeq \mathbf{0}$.

Let $f: \mathbb{C} \rightarrow \mathbb{C}$ be an entire analytic function with a power-series representation $f(x) \equiv \sum_{n=0}^{\infty} a_{n} x^{n}(x \in \mathbb{C})$. If all $a_{n}$ are real, the expression:

$$
f(A) \equiv \sum_{n=0}^{\infty} a_{n} A^{n}\left(A \in \mathbb{C}_{\text {Herm }}^{d \times d}\right)
$$

corresponds to a mapping from $\mathbb{C}_{H e r m}^{d \times d}$ to itself. We note that notions of convergence are as in [21]. The Spectral Mapping Theorem states that each eigenvalue of $f(A)$ is equal to $f(\lambda)$ with $\lambda \in \operatorname{spec}(A)$, i.e.,

$$
\operatorname{spec}(f(A))=f(\operatorname{spec}(A)) .
$$

In the sequel, we use the following lemma applied to the matrix exponential, to be defined shortly.

Lemma 7 (Lieb [29]). Let $f, g: \mathbb{R} \rightarrow \mathbb{R}$, and suppose there is a subset $S \subseteq \mathbb{R}$ with $f(a) \leqslant g(a)$ for all $a \in S$. If $A$ is a Hermitian matrix with all eigenvalues contained in $S$, then $f(A) \preceq g(A)$.

In our proofs, we make use of the matrix exponential, defined as $\exp (A)=\sum_{n=0}^{\infty} \frac{1}{n !} A^{n}$. From the Spectral Mapping Theorem we know that $\exp (A)$ is always positive definite when $A$ is Hermitian, and that $\exp (A)$ converges for all choices of $A$. By Lemma 7 , we have:

$$
\text { for any } A \in \mathbb{C}_{H e r m}^{d \times d}, I+A \preceq e^{A} \text {. }
$$

Moreover, we shall require brief use of the matrix logarithm. The matrix logarithm is defined as the functional inverse of the matrix exponential:

$$
\text { for any } A \in \mathbb{C}_{H e r m}^{d \times d}, \log \left(e^{A}\right):=A \text {. }
$$

This formula defines the logarithm of a positive definite matrix. In general, if $B=\exp (A)$, we say that $A$ is the logarithm of $B$. As our matrices will be Hermitian, it is sufficient for uniqueness of this function to require that the logarithm also be Hermitian. The matrix logarithm is monotone with respect to the positive semidefinite order (See [4]):

$$
\text { for any } A, B \in \mathbb{C}_{H e r m}^{d \times d} \text {, if } A \succ \mathbf{0}, B \succ \mathbf{0} \text { and } A \preceq B \text {, then } \log (A) \preceq \log (B) \text {. }
$$

Any notation not mentioned here pertaining to matrices is as in [21]. 


\section{$2.2 \quad$ Auxiliary concentration results}

We shall require the following concentration inequalities in order to prove our main theorems. Various matrix concentration inequalities have been derived by many authors, including Ahlswede and Winter [1], Cristofides and Markström [11], Oliveira [26], Gross [19], Recht [28], Tropp [29], and Chung and Radcliffe [8]. In [8], Chung and Radcliffe give a short proof for the following relatively simple version that is particularly suitable for random graphs.

Theorem 8 ([8]). Let $X_{1}, X_{2}, \ldots, X_{m}$ be independent random $n \times n$ Hermitian matrices. Moreover, assume that $\left\|X_{i}-\mathbb{E}\left(X_{i}\right)\right\| \leqslant c$ for all $i$. Let $X=\sum_{i=1}^{m} X_{i}$. Then for any $a>0$,

$$
\operatorname{Pr}(\|X-\mathbb{E}(X)\| \geqslant a) \leqslant 2 n \exp \left(-\frac{a^{2}}{2\left\|\sum_{i=1}^{m} \operatorname{Var}\left(X_{i}\right)\right\|+2 a c / 3}\right) .
$$

A strengthened version of Theorem 8 that we need for one of our proofs in Section 3, is as follows.

Theorem 9. Let $X_{1}, X_{2}, \ldots, X_{m}$ be independent random $n \times n$ Hermitian matrices. Moreover, assume that $\left\|X_{i}\right\| \leqslant c$ for all $i$. Let $X=\sum_{i=1}^{m} X_{i}$. Then

$$
\operatorname{Pr}\left(\lambda_{\max }(X) \geqslant a\right) \leqslant n \exp \left(-\frac{(a-\|\mathbb{E}(X)\|)^{2}}{2\left\|\sum_{i=1}^{m} \mathbb{E}\left(X_{i}^{2}\right)\right\|+\frac{2 c}{3}(a-\|\mathbb{E}(X)\|)}\right) \text { for } a>\|\mathbb{E}(X)\| \text {. }
$$

In particular,

$$
\operatorname{Pr}(\|X\| \geqslant a) \leqslant 2 n \exp \left(-\frac{(a-\|\mathbb{E}(X)\|)^{2}}{2\left\|\sum_{i=1}^{m} \mathbb{E}\left(X_{i}^{2}\right)\right\|+\frac{2 c}{3}(a-\|\mathbb{E}(X)\|)}\right) \text { for } a>\|\mathbb{E}(X)\| .
$$

Before we present our proof of Theorem 9, we will first show that Theorem 9 implies Theorem 8. For this purpose, let $X_{i}(1 \leqslant i \leqslant m)$ be as in Theorem 8. Let $X_{i}^{\prime}=X_{i}-\mathbb{E}\left(X_{i}\right)$ and $X^{\prime}=\sum_{i=1}^{m} X_{i}^{\prime}=X-\mathbb{E}(X)$. Then $\mathbb{E}\left(X^{\prime}\right)=\mathbf{0}$. From the hypothesis of Theorem 8 , we see that

$$
\left\|X_{i}^{\prime}\right\| \leqslant c \text { for all } i \in\{1, \ldots, m\} .
$$

We also have

$$
\begin{aligned}
\left\|\sum_{i=1}^{m} \mathbb{E}\left(X_{i}^{\prime 2}\right)\right\| & =\left\|\sum_{i=1}^{m} \mathbb{E}\left(X_{i}-\mathbb{E}\left(X_{i}\right)\right)^{2}\right\| \\
& =\left\|\sum_{i=1}^{m} \operatorname{Var}\left(X_{i}\right)\right\| .
\end{aligned}
$$

Applying Theorem 9, we get that for $a>0=\left\|\mathbb{E}\left(X^{\prime}\right)\right\|$,

$$
\operatorname{Pr}(\|X-\mathbb{E}(X)\| \geqslant a)=\operatorname{Pr}\left(\left\|X^{\prime}\right\| \geqslant a\right)
$$




$$
\begin{aligned}
& \leqslant 2 n \exp \left(-\frac{\left(a-\left\|\mathbb{E}\left(X^{\prime}\right)\right\|\right)^{2}}{2\left\|\sum_{i=1}^{m} \mathbb{E}\left(X_{i}^{\prime 2}\right)\right\|+\frac{2 c}{3}\left(a-\left\|\mathbb{E}\left(X^{\prime}\right)\right\|\right)}\right) \\
& =2 n \exp \left(-\frac{a^{2}}{2\left\|\sum_{i=1}^{m} \operatorname{Var}\left(X_{i}\right)\right\|+2 a c / 3}\right) .
\end{aligned}
$$

This shows that Theorem 9 implies Theorem 8.

\subsection{The proof of Theorem 9}

We are now going to prove Theorem 9. For our proof, we will rely on Lemma 7 and the following known result.

Lemma 10 ([29]). Consider a finite sequence $\left\{X_{i}\right\}_{i=1}^{m}$ of independent, random, Hermitian matrices. Then

$$
\mathbb{E}\left[\operatorname{Tr}\left(\exp \left(\sum_{i=1}^{m} \theta X_{i}\right)\right)\right] \leqslant \operatorname{Tr}\left(\exp \left(\sum_{i=1}^{m} \log \mathbb{E}\left(\exp \left(\theta X_{i}\right)\right)\right)\right) \text { for any constant } \theta \in \mathbb{R} .
$$

Proof of Theorem 9. We define

$$
g(x)=2 \sum_{k=2}^{\infty} \frac{x^{k-2}}{k !}=\frac{2\left(e^{x}-1-x\right)}{x^{2}}
$$

and first show the following facts about $g$, followed by short justifications for the statements.

- $g(0)=1$. In fact,

$$
g(0)=\lim _{x \rightarrow 0} \frac{2\left(e^{x}-1-x\right)}{x^{2}}=\lim _{x \rightarrow 0} \frac{2\left(e^{x}-1\right)}{2 x}=\lim _{x \rightarrow 0} \frac{e^{x}}{1}=1 .
$$

- $g(x)$ is monotone increasing for $x \geqslant 0$. Note that for $x \neq 0, g^{\prime}(x)=2 x^{-3}\left((x-2) e^{x}+\right.$ $x+2)$, and so it suffices to show that $h(x)=(x-2) e^{x}+x+2$ satisfies $h(x) \geqslant 0$ for all $x \in \mathbb{R}$. Clearly, $h(0)=0$ and $h^{\prime}(x)=(x-1) e^{x}+1$. Hence, $h^{\prime}(0)=0$ and $h^{\prime \prime}(x)=x e^{x}$, so $h^{\prime \prime}(x)<0$ for $x<0$ and $h^{\prime \prime}(x)>0$ for $x>0$. Therefore, $h^{\prime}(x)$ is monotone decreasing in $x \in(-\infty, 0]$ and $h^{\prime}(x)$ is monotone increasing in $x \in(0,+\infty)$. So, $h^{\prime}(x) \geqslant h^{\prime}(0)=0$ for all $x \in \mathbb{R}$. Thus, $h(x)$ is monotone increasing for all $x \in \mathbb{R}$. Indeed, $h(x) \geqslant h(0)=0$ for all $x \in \mathbb{R}$, as required.

- $g(x) \leqslant 1$ for $x<0$. In fact, $g^{\prime}(x)=2 x^{-3} h(x) \leqslant 0$ if $x<0$. So, the function $g$ is decreasing for $x<0$. Thus, $g(x) \leqslant g(0)=1$ for $x<0$. 
- for $x<3$, using $k$ ! $\geqslant 2 \cdot 3^{k-2}$, we obtain

$$
g(x)=2 \sum_{k=2}^{\infty} \frac{x^{k-2}}{k !} \leqslant \sum_{k=2}^{\infty} \frac{x^{k-2}}{3^{k-2}}=\frac{1}{1-x / 3} .
$$

Recalling that $g(x)$ is monotone increasing for $x \geqslant 0$, for $0<x \leqslant c$, we get $g(x) \leqslant g(c)$. Now let $X_{i}(1 \leqslant i \leqslant m)$ be as in the hypothesis of Theorem 9 .

Given a real constant $\theta>0$, we have $\left\|\theta X_{i}\right\| \leqslant \theta c$. Applying Lemma 7 , we obtain that $g\left(\theta X_{i}\right) \preceq g(\theta c) I$. Therefore, noting that $e^{x}=1+x+\frac{1}{2} x^{2} g(x)$, we have

$$
\begin{aligned}
e^{\theta X_{i}} & =I+\theta X_{i}+\frac{1}{2} \theta^{2} g\left(\theta X_{i}\right) X_{i}^{2} \\
& \preceq I+\theta X_{i}+\frac{1}{2} \theta^{2} g(\theta c) X_{i}^{2} .
\end{aligned}
$$

We now use that the expectation respects the positive semidefinite order (See [29]), i.e.,

$$
\text { for any } A, B \in \mathbb{C}_{H e r m}^{d \times d}, A \preceq B \text { almost surely implies } \mathbb{E} A \preceq \mathbb{E} B \text {. }
$$

Using (2.2), (2.7), and (2.8), we obtain

$$
\begin{aligned}
\mathbb{E}\left(e^{\theta X_{i}}\right) & \preceq \mathbb{E}\left(I+\theta X_{i}+\frac{1}{2} \theta^{2} g(\theta c) X_{i}^{2}\right) \\
& =I+\theta \mathbb{E}\left(X_{i}\right)+\frac{1}{2} \theta^{2} g(\theta c) \mathbb{E}\left(X_{i}^{2}\right) \\
& \preceq e^{\theta \mathbb{E}\left(X_{i}\right)+\frac{1}{2} \theta^{2} g(\theta c) \mathbb{E}\left(X_{i}^{2}\right)} .
\end{aligned}
$$

Next, we prove the following claim related to the trace of the matrix exponential.

Claim 1. For the given matrices $X_{i}$,

$$
\mathbb{E}\left[\operatorname{Tr}\left(\exp \left(\sum_{i=1}^{m} \theta X_{i}\right)\right)\right] \leqslant \operatorname{Tr}\left(\exp \left(\theta \mathbb{E}(X)+\frac{1}{2} \theta^{2} g(\theta c) \sum_{i=1}^{m} \mathbb{E}\left(X_{i}^{2}\right)\right)\right) .
$$

Proof of Claim 1. Here we work with the trace of the matrix exponential, $\operatorname{Tr}(\exp ): A \mapsto$ $\operatorname{Tr}(\exp (A))$. This trace exponential function is monotone with respect to the positive semidefinite order, i.e.,

$$
\forall A, B \in \mathbb{C}_{H e r m}^{d \times d}, A \preceq B \text { implies } \operatorname{Tr}(\exp (A)) \leqslant \operatorname{Tr}(\exp (B)) .
$$

See, e.g., [27], Section 2 for a short proof of this fact. Now, using Lemma 10, (2.3), (2.4), (2.9) and (2.11), we obtain

$$
\mathbb{E}\left[\operatorname{Tr}\left(\exp \left(\sum_{i=1}^{m} \theta X_{i}\right)\right)\right] \leqslant \operatorname{Tr}\left(\exp \left(\sum_{i=1}^{m} \log \mathbb{E}\left(\exp \left(\theta X_{i}\right)\right)\right)\right)
$$




$$
\begin{aligned}
& \leqslant \operatorname{Tr}\left(\exp \left(\sum_{i=1}^{m} \log e^{\theta \mathbb{E}\left(X_{i}\right)+\frac{1}{2} \theta^{2} g(\theta c) \mathbb{E}\left(X_{i}^{2}\right)}\right)\right) \\
& =\operatorname{Tr}\left(\exp \left(\sum_{i=1}^{m}\left(\theta \mathbb{E}\left(X_{i}\right)+\frac{1}{2} \theta^{2} g(\theta c) \mathbb{E}\left(X_{i}^{2}\right)\right)\right)\right) \\
& =\operatorname{Tr}\left(\exp \left(\theta \mathbb{E}(X)+\frac{1}{2} \theta^{2} g(\theta c) \sum_{i=1}^{m} \mathbb{E}\left(X_{i}^{2}\right)\right)\right),
\end{aligned}
$$

as required.

Note that $\exp \left(\theta \lambda_{\max }(X)\right)$ is a random variable. Then, by Markov's inequality, for any real constant $a>0$,

$$
\operatorname{Pr}\left(\lambda_{\max }(X) \geqslant a\right)=\operatorname{Pr}\left(e^{\theta \lambda_{\max }(X)} \geqslant e^{\theta a}\right) \leqslant e^{-\theta a} \mathbb{E}\left(e^{\theta \lambda_{\max }(X)}\right)
$$

By (2.1), for any $s \geqslant 0$, and for any $A \in \mathbb{C}_{H e r m}^{d \times d}$, the largest eigenvalue of $e^{s A}$ is $e^{s \lambda_{\max }(A)}$ and all eigenvalues of $e^{s A}$ are nonnegative. Hence,

$$
\exp \left(s \lambda_{\max }(A)\right)=\lambda_{\max }(\exp (s A)) \leqslant \operatorname{Tr}(\exp (s A)) .
$$

We need two more inequalities from matrix analysis, where the first one is usually referred to as the Golden-Thompson inequality (See, e.g., [3]), and the second one can be found, e.g., in [31].

$$
\forall d \in\{1,2,3, \ldots\}, \text { and any } A, B \in \mathbb{C}_{H e r m}^{d \times d}, \operatorname{Tr}\left(e^{A+B}\right) \leqslant \operatorname{Tr}\left(e^{A} e^{B}\right) .
$$

If $A$ and $B$ are $n \times n$ positive semidefinite Hermitian matrices, then

$$
0 \leqslant \operatorname{Tr}(A \cdot B) \leqslant \operatorname{Tr}(A) \cdot \lambda_{\max }(B) \leqslant \operatorname{Tr}(A) \cdot \operatorname{Tr}(B) .
$$

Now, given a real constant $a>\|\mathbb{E}(X)\|$, for every real constant $\theta>0$, using (2.10), (2.12), (2.13), (2.14), and (2.15), we obtain

$$
\begin{aligned}
\operatorname{Pr}\left(\lambda_{\max }(X) \geqslant a\right) & \leqslant e^{-\theta a} \mathbb{E}\left(e^{\theta \lambda_{\max }(X)}\right) \\
& \leqslant e^{-\theta a} \mathbb{E}(\operatorname{Tr}(\exp (\theta X)) \\
& =e^{-\theta a} \mathbb{E}\left[\operatorname{Tr}\left(\exp \left(\sum_{i=1}^{m} \theta X_{i}\right)\right)\right] \\
& \leqslant e^{-\theta a} \operatorname{Tr}\left[\exp \left(\theta \mathbb{E}(X)+\frac{1}{2} \theta^{2} g(\theta c) \sum_{i=1}^{m} \mathbb{E}\left(X_{i}^{2}\right)\right)\right] \\
& \leqslant e^{-\theta a} \operatorname{Tr}\left[\exp (\theta \mathbb{E}(X)) \cdot \exp \left(\frac{1}{2} \theta^{2} g(\theta c) \sum_{i=1}^{m} \mathbb{E}\left(X_{i}^{2}\right)\right)\right] \\
& \leqslant e^{-\theta a} \lambda_{\max }(\exp (\theta \mathbb{E}(X))) \cdot \operatorname{Tr}\left[\exp \left(\frac{1}{2} \theta^{2} g(\theta c) \sum_{i=1}^{m} \mathbb{E}\left(X_{i}^{2}\right)\right)\right]
\end{aligned}
$$




$$
\begin{aligned}
& \leqslant e^{-\theta a} \lambda_{\max }(\exp (\theta \mathbb{E}(X))) \cdot n \lambda_{\max }\left[\exp \left(\frac{1}{2} \theta^{2} g(\theta c) \sum_{i=1}^{m} \mathbb{E}\left(X_{i}^{2}\right)\right)\right] \\
& =n e^{-\theta a}\left(\exp \left(\theta \lambda_{\max }(\mathbb{E}(X))\right)\right) \cdot \exp \left(\frac{1}{2} \theta^{2} g(\theta c) \lambda_{\max }\left(\sum_{i=1}^{m} \mathbb{E}\left(X_{i}^{2}\right)\right)\right) \\
& =n \exp \left(-\theta a+\theta \lambda_{\max }(\mathbb{E}(X))+\frac{1}{2} \theta^{2} g(\theta c) \lambda_{\max }\left(\sum_{i=1}^{m} \mathbb{E}\left(X_{i}^{2}\right)\right)\right) \\
& \leqslant n \exp \left(-\theta a+\theta\|\mathbb{E}(X)\|+\frac{1}{2} \theta^{2} g(\theta c)\left\|\sum_{i=1}^{m} \mathbb{E}\left(X_{i}^{2}\right)\right\|\right)
\end{aligned}
$$

The final inequality holds as $\|\mathbb{E}(X)\| \geqslant \lambda_{\max }(\mathbb{E}(X))$ and $\left\|\sum_{i=1}^{m} \mathbb{E}\left(X_{i}^{2}\right)\right\| \geqslant \lambda_{\max }\left(\sum_{i=1}^{m} \mathbb{E}\left(X_{i}^{2}\right)\right)$.

Recall that we assume $a>\|\mathbb{E}(X)\|$. Now take $\theta=\frac{a-\|\mathbb{E}(X)\|}{\left\|\sum_{i=1}^{m} \mathbb{E}\left(X_{i}^{2}\right)\right\|+\frac{c}{3}(a-\|\mathbb{E}(X)\|)}$. Then, clearly $\theta c<3$. Using (2.6), we obtain

$$
\begin{aligned}
& \operatorname{Pr}\left(\lambda_{\max }(X) \geqslant a\right) \leqslant n \exp \left(-\theta a+\theta\|\mathbb{E}(X)\|+\frac{1}{2} \theta^{2} g(\theta c)\left\|\sum_{i=1}^{m} \mathbb{E}\left(X_{i}^{2}\right)\right\|\right) \\
& \leqslant n \exp \left(-\theta a+\theta\|\mathbb{E}(X)\|+\frac{\theta^{2}\left\|\sum_{i=1}^{m} \mathbb{E}\left(X_{i}^{2}\right)\right\|}{2\left(1-\frac{\theta c}{3}\right)}\right) \\
& =n \exp \left(-\theta\left[a-\|\mathbb{E}(X)\|-\frac{\theta\left\|\sum_{i=1}^{m} \mathbb{E}\left(X_{i}^{2}\right)\right\|}{2\left(1-\frac{\theta c}{3}\right)}\right]\right) \\
& =n \exp \left(-\theta\left[a-\|\mathbb{E}(X)\|-\frac{\theta\left\|\sum_{i=1}^{m} \mathbb{E}\left(X_{i}^{2}\right)\right\|}{2\left(1-\frac{a-\|\mathbb{E}(X)\|}{\left\|\sum_{i=1}^{m} \mathbb{E}\left(X_{i}^{2}\right)\right\|+\frac{c}{3}(a-\|\mathbb{E}(X)\|)} \cdot \frac{c}{3}\right)}\right]\right) \\
& =n \exp \left(-\theta\left[a-\|\mathbb{E}(X)\|-\frac{\theta\left\|\sum_{i=1}^{m} \mathbb{E}\left(X_{i}^{2}\right)\right\|}{\left.\left.\frac{2\left\|\sum_{i=1}^{m} \mathbb{E}\left(X_{i}^{2}\right)\right\|}{\left\|\sum_{i=1}^{m} \mathbb{E}\left(X_{i}^{2}\right)\right\|+\frac{c}{3}(a-\|\mathbb{E}(X)\|)}\right]\right)}\right.\right. \\
& =n \exp \left(-\theta\left[a-\|\mathbb{E}(X)\|-\frac{\theta}{2}\left(\left\|\sum_{i=1}^{m} \mathbb{E}\left(X_{i}^{2}\right)\right\|+\frac{c}{3}(a-\|\mathbb{E}(X)\|)\right)\right]\right) \\
& =n \exp \left(-\theta\left[a-\|\mathbb{E}(X)\|-\frac{1}{2}(a-\|\mathbb{E}(X)\|)\right]\right) \\
& =n \exp \left(-\frac{\theta}{2}(a-\|\mathbb{E}(X)\|)\right) \\
& =n \exp \left(-\frac{(a-\|\mathbb{E}(X)\|)^{2}}{2\left\|\sum_{i=1}^{m} \mathbb{E}\left(X_{i}^{2}\right)\right\|+\frac{2 c}{3}(a-\|\mathbb{E}(X)\|)}\right) \text {. }
\end{aligned}
$$

This proves the first statement of Theorem 9. To obtain the norm bound (2.5) in the 
second statement of Theorem 9, recall that

$$
\text { for any } Y \in \mathbb{C}_{\text {Herm }}^{n \times n},\|Y\|=\max \left\{\lambda_{\max }(Y),-\lambda_{\min }(Y)\right\}=\max \left\{\lambda_{\max }(Y), \lambda_{\max }(-Y)\right\} .
$$

Using this, we next apply the inequality (2.16) to the sequence $\left\{-X_{i}\right\}$, i.e., we replace the sequence $\left\{X_{i}\right\}$ by the sequence $\left\{-X_{i}\right\}$ in the above inequality (2.16). We obtain

$$
\begin{aligned}
\operatorname{Pr}\left(\lambda_{\max }(-X) \geqslant a\right) & \leqslant n \exp \left(-\frac{(a-\|\mathbb{E}(-X)\|)^{2}}{2\left\|\sum_{i=1}^{m} \mathbb{E}\left(\left(-X_{i}\right)^{2}\right)\right\|+\frac{2 c}{3}(a-\|\mathbb{E}(-X)\|)}\right) \\
& =n \exp \left(-\frac{(a-\|\mathbb{E}(X)\|)^{2}}{2\left\|\sum_{i=1}^{m} \mathbb{E}\left(X_{i}^{2}\right)\right\|+\frac{2 c}{3}(a-\|\mathbb{E}(X)\|)}\right) .
\end{aligned}
$$

Applying the union bound to the estimates for $\lambda_{\max }(X)$ and $-\lambda_{\min }(X)$, we obtain

$$
\operatorname{Pr}(\|X\| \geqslant a) \leqslant 2 n \exp \left(-\frac{(a-\|\mathbb{E}(X)\|)^{2}}{2\left\|\sum_{i=1}^{m} \mathbb{E}\left(X_{i}^{2}\right)\right\|+\frac{2 c}{3}(a-\|\mathbb{E}(X)\|)}\right) .
$$

This completes the proof of Theorem 9 .

\section{Proofs of Theorem 1 and Theorem 4}

In this section, we provide proofs for the two main theorems of this paper, using Theorem 8 , Theorem 9 , and a number of other known results.

\subsection{The proof of Theorem 1}

Before presenting our proof of Theorem 1, we recall one more known result that will be used in the sequel of the paper.

Lemma 11 ([21]). Let $M=\left(m_{i j}\right)$ be an $n \times n$ matrix. Then

$$
\rho(M) \leqslant \min \left\{\max _{1 \leqslant i \leqslant n} \sum_{j=1}^{n}\left|m_{i j}\right|, \max _{1 \leqslant j \leqslant n} \sum_{i=1}^{n}\left|m_{i j}\right|\right\} .
$$

Proof of Theorem 1. Let $\widehat{G}_{n}\left(p_{i j}\right)$ and $H_{n}=\left(h_{i j}\right)$ be defined as before, and let $\Delta=$ $\Delta\left(\Gamma\left(\widehat{G}_{n}\left(p_{i j}\right)\right)\right)$. Recall that this implies that $\Delta=\max _{1 \leqslant i \leqslant n} \sum_{j=1}^{n}\left(p_{i j}+p_{j i}-p_{i j} p_{j i}\right)$.

For the indices $i$ and $j$ with $1 \leqslant i, j \leqslant n$, let $H^{i j}$ be the $n \times n$ matrix with a 1 in the $(i, j)$-th position and a 0 everywhere else. Recall that $h_{i j}$ takes value 1 with probability $p_{i j} p_{j i}$, value $\mathbf{i}$ with probability $p_{i j}\left(1-p_{j i}\right)$, value $-\mathbf{i}$ with probability $\left(1-p_{i j}\right) p_{j i}$, and value 0 with probability $\left(1-p_{i j}\right)\left(1-p_{j i}\right)$. So, $h_{j i}=\overline{h_{i j}}$, i.e., $h_{j i}$ is the complex conjugate of $h_{i j}$. Take $X_{i j}=h_{i j} H^{i j}+h_{j i} H^{j i}=h_{i j} H^{i j}+\overline{h_{i j}} H^{j i}$. Then, $H_{n}=\sum_{1 \leqslant i<j \leqslant n} X_{i j}$. Now, we can apply Theorem 9 to $H_{n}$ if we derive a suitable upper bound $c$ on $\left\|X_{i j}\right\|$. Note that $X_{i j}$ 
$(1 \leqslant i<j \leqslant n)$ are independent random $n \times n$ Hermitian matrices, and that, with the choice $c=1$,

$$
\left\|X_{i j}\right\|=\left\|h_{i j} H^{i j}+\overline{h_{i j}} H^{j i}\right\|=\left|h_{i j}\right|<1=c .
$$

Before applying Theorem 9, we first perform some additional calculations in order to obtain upper bounds for $\left\|\sum_{1 \leqslant i<j \leqslant n} \mathbb{E}\left(X_{i j}^{2}\right)\right\|$ and $\left\|\mathbb{E} H_{n}\right\|$.

For all $1 \leqslant i<j \leqslant n$, we have

$$
\begin{aligned}
\mathbb{E}\left(X_{i j}^{2}\right) & =\mathbb{E}\left(h_{i j} H^{i j}+\overline{h_{i j}} H^{j i}\right)^{2} \\
& =\mathbb{E}\left[h_{i j} \cdot \overline{h_{i j}}\right]\left(H^{i i}+H^{j j}\right) \\
& =\mathbb{E}\left[\left|h_{i j}\right|^{2}\right]\left(H^{i i}+H^{j j}\right) \\
& =\left(p_{i j}+p_{j i}-p_{i j} p_{j i}\right)\left(H^{i i}+H^{j j}\right) .
\end{aligned}
$$

We set $p_{i i}=0$. Then,

$$
\begin{aligned}
\left\|\sum_{1 \leqslant i<j \leqslant n} \mathbb{E}\left(X_{i j}^{2}\right)\right\| & =\left\|\sum_{i=1}^{n}\left(\sum_{j=1}^{n}\left(p_{i j}+p_{j i}-p_{i j} p_{j i}\right)\right) H^{i i}\right\| \\
& =\max _{i=1, \ldots, n} \sum_{j=1}^{n}\left(p_{i j}+p_{j i}-p_{i j} p_{j i}\right) \\
& =\Delta .
\end{aligned}
$$

Recall that $\left(\mathbb{E} H_{n}\right)_{i j}=\mathbb{E} h_{i j}=p_{i j} p_{j i}+\mathbf{i}\left(p_{i j}-p_{j i}\right)$, and in particular, $\mathbb{E} H_{n}$ is a Hermitian matrix. So, $\left\|\mathbb{E} H_{n}\right\|=\rho\left(\mathbb{E} H_{n}\right)$. By Lemma 11, we have

$$
\begin{aligned}
\left\|\mathbb{E} H_{n}\right\| & =\rho\left(\mathbb{E} H_{n}\right) \\
& \leqslant \min \left\{\max _{1 \leqslant i \leqslant n} \sum_{j=1}^{n}\left|\mathbb{E} h_{i j}\right|, \max _{1 \leqslant j \leqslant n} \sum_{i=1}^{n}\left|\mathbb{E} h_{i j}\right|\right\} \\
& \leqslant \max _{1 \leqslant i \leqslant n} \sum_{j=1}^{n} \sqrt{p_{i j}^{2} p_{j i}^{2}+\left(p_{i j}-p_{j i}\right)^{2}} .
\end{aligned}
$$

Now, we take $a=\left\|\mathbb{E} H_{n}\right\|+\sqrt{4 \Delta \ln (2 n / \epsilon)}$. By the assumption that $\Delta>\frac{4}{9} \ln (2 n / \epsilon)$, we obtain that $a-\left\|\mathbb{E} H_{n}\right\|<3 \Delta$. Applying Theorem 9, and using $c=1$, we get

$$
\begin{aligned}
\operatorname{Pr}\left(\left\|H_{n}\right\| \geqslant a\right) & \leqslant 2 n \exp \left(-\frac{\left(a-\left\|\mathbb{E} H_{n}\right\|\right)^{2}}{2\left\|\sum_{1 \leqslant i<j \leqslant n} \mathbb{E}\left(X_{i j}^{2}\right)\right\|+\frac{2 c}{3}\left(a-\left\|\mathbb{E} H_{n}\right\|\right)}\right) \\
& \leqslant 2 n \exp \left(-\frac{4 \Delta \ln (2 n / \epsilon)}{4 \Delta}\right) \\
& =\epsilon .
\end{aligned}
$$

Thus, with probability at least $1-\epsilon$, we have that for all $1 \leqslant i \leqslant n$,

$$
\left|\lambda_{i}\left(H_{n}\right)\right| \leqslant\left\|H_{n}\right\|
$$




$$
\begin{aligned}
& \leqslant a \\
& =\left\|\mathbb{E} H_{n}\right\|+\sqrt{4 \Delta \ln (2 n / \epsilon)} .
\end{aligned}
$$

This completes the proof of Theorem 1 .

\subsection{The proof of Theorem 4}

Before presenting the proof of Theorem 4, we recall some known results that will be used in the sequel of the paper.

Lemma 12 (Weyl [30]). Let $X, Y$ and $Z$ be $n \times n$ Hermitian matrices such that $X=Y+$ $Z$. Suppose that $X, Y, Z$ have eigenvalues $\lambda_{1}(X) \geqslant \cdots \geqslant \lambda_{n}(X), \lambda_{1}(Y) \geqslant \cdots \geqslant \lambda_{n}(Y)$, $\lambda_{1}(Z) \geqslant \cdots \geqslant \lambda_{n}(Z)$, respectively. Then for $i=1,2, \ldots, n$ the following inequalities hold:

$$
\lambda_{i}(Y)+\lambda_{n}(Z) \leqslant \lambda_{i}(X) \leqslant \lambda_{i}(Y)+\lambda_{1}(Z) .
$$

We also use the following concentration result. It involves a variation on the Chernoff bound, and can, e.g., be found as Lemma A in [6].

Lemma 13. Let $X_{1}, X_{2}, \ldots, X_{m}$ be independent random variables satisfying $\left|X_{i}\right| \leqslant c$ for all $i$. Let $X=\sum_{i=1}^{m} X_{i}$. Then for any $a>0$,

$$
\operatorname{Pr}(|X-\mathbb{E}(X)| \geqslant a) \leqslant \exp \left(-\frac{a^{2}}{2 \sum_{i=1}^{m} \operatorname{Var}\left(X_{i}\right)+2 a c / 3}\right) .
$$

Finally, we will use the following min-max result due to Courant-Fischer (Theorem 4.2 .11 in [21]).

Lemma 14. Let $A$ be an $n \times n$ Hermitian matrix with eigenvalues $\lambda_{1} \leqslant \lambda_{2} \leqslant \ldots \leqslant \lambda_{n}$, and let $k$ be an integer with $1 \leqslant k \leqslant n$. Then

$$
\lambda_{k}=\min _{w_{1}, w_{2}, \ldots, w_{n-k} \in \mathbb{C}^{n}} \max _{\substack{x \neq 0 \\ x \perp w_{1}, w_{2}, \ldots, w_{n-k}}} \frac{x^{*} A x}{x^{*} x} .
$$

Let $G=(V(G), E(G), A(G))$ be a mixed graph of order $n$. For brevity, we write $D$ for $D(G), L$ for $L(G)$ and $\mathcal{L}$ for $\mathcal{L}(G)$. Hence, $\mathcal{L}=I-D^{-\frac{1}{2}} H D^{-\frac{1}{2}}=D^{-1 / 2} L D^{-1 / 2}$. We are first going to show that $\mathcal{L}$ is positive semidefinite, by deriving an alternative expression for $\frac{x^{*} \mathcal{L} x}{x^{*} x}$ for an arbitrary nonzero complex $n \times 1$ column vector $x$. After that, we are going to expand the alternative expression in order to obtain an upper bound for the eigenvalues of $\mathcal{L}$, using Lemma 14.

In the following expansion, $y=D^{-1 / 2} x, N\left(v_{i}\right)$ denotes the neighborhood of $v_{i}$ in the underlying graph $\Gamma(G)$, and $\sum_{e=v_{i} v_{j}}$ denotes the sum over all unordered pairs $\left\{v_{i}, v_{j}\right\}$ for which $v_{i}$ and $v_{j}$ are adjacent in $\Gamma(G)$.

$$
\frac{x^{*} \mathcal{L} x}{x^{*} x}=\frac{x^{*} D^{-1 / 2} L D^{-1 / 2} x}{x^{*} x}
$$




$$
\begin{aligned}
& =\frac{y^{*} L y}{\left(D^{1 / 2} y\right)^{*}\left(D^{1 / 2} y\right)} \\
& =\frac{y^{*} L y}{y^{*} D y} \\
& =\frac{\left(y_{1}^{*}, y_{2}^{*}, \ldots, y_{n}^{*}\right)\left(\begin{array}{cccc}
d_{1} & -h_{12} & \cdots & -h_{1 n} \\
-h_{21} & d_{2} & \cdots & -h_{2 n} \\
\vdots & \vdots & \ddots & \vdots \\
-h_{n 1} & -h_{n 2} & \cdots & d_{n}
\end{array}\right)\left(\begin{array}{c}
y_{1} \\
y_{2} \\
\vdots \\
y_{n}
\end{array}\right)}{\left(y_{1}^{*}, y_{2}^{*}, \ldots, y_{n}^{*}\right)\left(\begin{array}{cccc}
d_{1} & & & \\
& d_{2} & & \\
& & \ddots & \\
& & & d_{n}
\end{array}\right)\left(\begin{array}{c}
y_{1} \\
y_{2} \\
\vdots \\
y_{n}
\end{array}\right)} \\
& =\frac{\sum_{v_{i} \in V(G)} d_{i}\left|y_{i}\right|^{2}-\sum_{v_{i} \neq v_{j}} h_{i j} y_{i}^{*} y_{j}}{\sum_{v_{i} \in V(G)} d_{i}\left|y_{i}\right|^{2}} \\
& =\frac{\sum_{v_{i}} \sum_{v_{j} \in N\left(v_{i}\right)}\left|y_{j}\right|^{2}-\sum_{v_{i} \neq v_{j}} h_{i j} y_{i}^{*} y_{j}}{\sum_{v_{i} \in V(G)} d_{i}\left|y_{i}\right|^{2}} \\
& =\frac{\sum_{e=v_{i} v_{j}}\left(\left|y_{i}\right|^{2}+\left|y_{j}\right|^{2}\right)-\sum_{e=v_{i} v_{j}}\left(h_{i j} y_{i}^{*} y_{j}+h_{j i} y_{i} y_{j}^{*}\right)}{\sum_{v_{i} \in V(G)} d_{i}\left|y_{i}\right|^{2}} \\
& =\frac{\sum_{e=v_{i} v_{j}}\left(\left|y_{i}\right|^{2}+\left|y_{j}\right|^{2}\right)-\sum_{e=v_{i} v_{j}}\left(h_{i j} y_{i}^{*} y_{j}+h_{i j}^{*} y_{i} y_{j}^{*}\right)}{\sum_{v_{i} \in V(G)} d_{i}\left|y_{i}\right|^{2}} \\
& =\frac{\sum_{e=v_{i} v_{j}}\left(y_{i}-h_{i j} y_{j}\right)\left(y_{i}^{*}-h_{i j}^{*} y_{j}^{*}\right)}{\sum_{v_{i} \in V(G)} d_{i}\left|y_{i}\right|^{2}} \\
& =\frac{\sum_{e=v_{i} v_{j}}\left(y_{i}-h_{i j} y_{j}\right)\left(y_{i}-h_{i j} y_{j}\right)^{*}}{\sum_{v_{i} \in V(G)} d_{i}\left|y_{i}\right|^{2}} \\
& =\frac{\sum_{e=v_{i} v_{j}}\left|y_{i}-h_{i j} y_{j}\right|^{2}}{\sum_{v_{i} \in V(G)} d_{i}\left|y_{i}\right|^{2}} \text {. }
\end{aligned}
$$

Before we continue our calculations, we note that the derived expression for $\frac{x^{*} \mathcal{L} x}{x^{*} x} \mathrm{im}$ plies that $\mathcal{L}$ is positive semidefinite. Next, we are going to expand the obtained expression for $\frac{x^{*} \mathcal{L} x}{x^{*} x}$, using the known fact that

$$
|f(x)-f(y)|^{2} \leqslant 2\left(|f(x)|^{2}+|f(y)|^{2}\right),
$$

where equality holds if and only if $f(x)=-f(y)$.

We split $\sum_{e=v_{i} v_{j}}$ in the above expression by distinguishing undirected edges (or pairs of oppositely oriented arcs), denoted as $v_{i} \leftrightarrow v_{j}$, and arcs, denoted as $v_{i} \rightarrow v_{j}$ if the orientation is from $v_{i}$ to $v_{j}$, and as $v_{i} \leftarrow v_{j}$ if the orientation is from $v_{j}$ to $v_{i}$. Adopting 
this notation, and using (3.1), we obtain

$$
\begin{aligned}
\sum_{e=v_{i} v_{j}}\left|y_{i}-h_{i j} y_{j}\right|^{2} & =\sum_{v_{i} \leftrightarrow v_{j}}\left|y_{i}-y_{j}\right|^{2}+\sum_{v_{i} \rightarrow v_{j} \text { or } v_{i} \leftarrow v_{j}}\left|y_{i}-h_{i j} y_{j}\right|^{2} \\
& \leqslant \sum_{v_{i} \leftrightarrow v_{j}} 2\left(\left|y_{i}\right|^{2}+\left|y_{j}\right|^{2}\right)+\sum_{v_{i} \rightarrow v_{j} \text { or } v_{i} \leftarrow v_{j}} 2\left(\left|y_{i}\right|^{2}+\left|h_{i j} y_{j}\right|^{2}\right) \\
& =\sum_{v_{i} \leftrightarrow v_{j}} 2\left(\left|y_{i}\right|^{2}+\left|y_{j}\right|^{2}\right)+\sum_{v_{i} \rightarrow v_{j} \text { or } v_{i} \leftarrow v_{j}} 2\left(\left|y_{i}\right|^{2}+\left|y_{j}\right|^{2}\right) \\
& =2\left(\sum_{v_{i} \leftrightarrow v_{j}}\left(\left|y_{i}\right|^{2}+\left|y_{j}\right|^{2}\right)+\sum_{v_{i} \rightarrow v_{j} \text { or } v_{i} \leftarrow v_{j}}\left(\left|y_{i}\right|^{2}+\left|y_{j}\right|^{2}\right)\right) .
\end{aligned}
$$

We also obtain

$$
\begin{aligned}
\sum_{v_{i} \in V(G)} d_{i}\left|y_{i}\right|^{2} & =\sum_{v_{i}} \sum_{v_{j} \in N\left(v_{i}\right)}\left|y_{j}\right|^{2} \\
& =\sum_{e=v_{i} v_{j}}\left(\left|y_{i}\right|^{2}+\left|y_{j}\right|^{2}\right) \\
& =\sum_{v_{i} \leftrightarrow v_{j}}\left(\left|y_{i}\right|^{2}+\left|y_{j}\right|^{2}\right)+\sum_{v_{i} \rightarrow v_{j} \text { or } v_{i} \leftarrow v_{j}}\left(\left|y_{i}\right|^{2}+\left|y_{j}\right|^{2}\right) .
\end{aligned}
$$

Therefore, using the latter two expressions and applying Lemma 14, we get the following upper bound on the eigenvalues of $\mathcal{L}$.

$$
\lambda_{i}(\mathcal{L}) \leqslant \sup _{x} \frac{\sum_{e=v_{i} v_{j}}\left|y_{i}-h_{i j} y_{j}\right|^{2}}{\sum_{v_{i} \in V(G)} d_{i}\left|y_{i}\right|^{2}} \leqslant 2 .
$$

This shows that the normalized Hermitian Laplacian spectrum is in $[0,2]$, and hence that $\|I-\mathcal{L}\| \leqslant 1$. We will use this conclusion near the end of the proof of Theorem 4 . We now have all the ingredients to present our proof of Theorem 4.

Proof of Theorem 4. Let $\widehat{G}_{n}\left(p_{i j}\right)$ and $H_{n}=\left(h_{i j}\right)$ be defined as before, and let $\delta=$ $\delta\left(\Gamma\left(\widehat{G}_{n}\left(p_{i j}\right)\right)\right)$. Recall that this implies that $\delta=\min _{1 \leqslant i \leqslant n} \sum_{j=1}^{n}\left(p_{i j}+p_{j i}-p_{i j} p_{j i}\right)$.

For each vertex $v_{i}$ of $\widehat{G}_{n}\left(p_{i j}\right)$, we let $d_{i}$ denote the degree of $v_{i}$ in the underlying graph $\Gamma\left(\widehat{G}_{n}\left(p_{i j}\right)\right)$, and we use $t_{i}=\mathbb{E}\left(d_{i}\right)$ to denote the expected degree of $v_{i}$, so $\mathbb{E} D_{n}=$ $\operatorname{diag}\left(\mathbb{E}\left(d_{1}\right), \mathbb{E}\left(d_{2}\right), \ldots, \mathbb{E}\left(d_{n}\right)\right)=\operatorname{diag}\left(t_{1}, t_{2}, \ldots, t_{n}\right)$. This means that the matrix $\widetilde{\mathcal{L}_{n}}=$ $I_{n}-\left(\mathbb{E} D_{n}\right)^{-1 / 2}\left(\mathbb{E} H_{n}\right)\left(\mathbb{E} D_{n}\right)^{-1 / 2}$ can be considered as the "expected Laplacian matrix" of $\widehat{G}_{n}\left(p_{i j}\right)$. Let $C_{n}=I_{n}-\left(\mathbb{E} D_{n}\right)^{-1 / 2} H_{n}\left(\mathbb{E} D_{n}\right)^{-1 / 2}$. Then, clearly

$$
\left\|\mathcal{L}_{n}-\widetilde{\mathcal{L}_{n}}\right\| \leqslant\left\|C_{n}-\widetilde{\mathcal{L}_{n}}\right\|+\left\|\mathcal{L}_{n}-C_{n}\right\|=\left\|\widetilde{\mathcal{L}_{n}}-C_{n}\right\|+\left\|C_{n}-\mathcal{L}_{n}\right\| .
$$

In the next stages, we derive bounds for each of the last two terms separately.

We first consider $\widetilde{\mathcal{L}_{n}}-C_{n}=\left(\mathbb{E} D_{n}\right)^{-1 / 2}\left(H_{n}-\mathbb{E} H_{n}\right)\left(\mathbb{E} D_{n}\right)^{-1 / 2}$. Let

$$
Y_{i j}=\left(\mathbb{E} D_{n}\right)^{-1 / 2}\left[\left(h_{i j}-\mathbb{E} h_{i j}\right) H^{i j}+\left(h_{j i}-\mathbb{E} h_{j i}\right) H^{j i}\right]\left(\mathbb{E} D_{n}\right)^{-1 / 2}
$$




$$
=\frac{\left(h_{i j}-\mathbb{E} h_{i j}\right) H^{i j}+\left(\overline{h_{i j}}-\mathbb{E} \overline{h_{i j}}\right) H^{j i}}{\sqrt{t_{i} t_{j}}} .
$$

Then, $\widetilde{\mathcal{L}_{n}}-C_{n}=\sum_{1 \leqslant i<j \leqslant n} Y_{i j}$. We are going to apply Theorem 8 to obtain an upper bound for $\left\|\widetilde{\mathcal{L}_{n}}-C_{n}\right\|$. Before we can do so, we have to perform some preliminary calculations in order to obtain an upper bound $c_{0}$ for $\left\|Y_{i j}-\mathbb{E}\left(Y_{i j}\right)\right\|$, and a suitable upper bound for $\left\|\sum_{1 \leqslant i<j \leqslant n} \operatorname{Var}\left(Y_{i j}\right)\right\|$. First of all, note that for all $1 \leqslant i<j \leqslant n$,

$$
\mathbb{E}\left(Y_{i j}\right)=\mathbb{E}\left[\frac{\left(h_{i j}-\mathbb{E} h_{i j}\right) H^{i j}+\left(\overline{h_{i j}}-\mathbb{E} \overline{h_{i j}}\right) H^{j i}}{\sqrt{t_{i} t_{j}}}\right]=\mathbf{0 .}
$$

We set $\mathbb{E}\left(Y_{i i}\right)=\mathbf{0}$. Then,

$$
\begin{aligned}
& \left\|Y_{i j}-\mathbb{E}\left(Y_{i j}\right)\right\|=\left\|Y_{i j}\right\| \\
& =\frac{\left\|\left(h_{i j}-\mathbb{E} h_{i j}\right) H^{i j}+\left(\overline{h_{i j}}-\mathbb{E} \overline{h_{i j}}\right) H^{j i}\right\|}{\sqrt{t_{i} t_{j}}} \\
& =\frac{\left\|\left(h_{i j}-\mathbb{E} h_{i j}\right) H^{i j}+\left(\overline{h_{i j}-\mathbb{E} h_{i j}}\right) H^{j i}\right\|}{\sqrt{t_{i} t_{j}}} \\
& =\frac{\left|h_{i j}-\mathbb{E} h_{i j}\right|}{\sqrt{t_{i} t_{j}}} \\
& =\frac{\left|h_{i j}-\left[p_{i j} p_{j i}+\mathbf{i}\left(p_{i j}-p_{j i}\right)\right]\right|}{\sqrt{t_{i} t_{j}}} \\
& = \begin{cases}\frac{\sqrt{\left(1-p_{i j} p_{j i}\right)^{2}+\left(p_{i j}-p_{j i}\right)^{2}}}{\sqrt{t_{i} t_{j}}}, & \text { for } h_{i j}=1, \\
\frac{\sqrt{\left(p_{i j} p_{j i}\right)^{2}+\left(1-\left(p_{i j}-p_{j i}\right)\right)^{2}}}{\sqrt{t_{i} t_{j}}}, & \text { for } h_{i j}=\mathbf{i}, \\
\frac{\sqrt{\left(p_{i j} p_{j i}\right)^{2}+\left(1+\left(p_{i j}-p_{j i}\right)\right)^{2}}}{\sqrt{t_{i} t_{j}}}, & \text { for } h_{i j}=-\mathbf{i}, \\
\frac{\sqrt{\left(p_{i j} p_{j i}\right)^{2}+\left(p_{i j}-p_{j i}\right)^{2}}}{\sqrt{t_{i} t_{j}}}, & \text { for } h_{i j}=0 .\end{cases} \\
& \leqslant \begin{cases}\frac{\sqrt{2}}{\sqrt{t_{i} t_{j}}}, & \text { for } h_{i j}=1, \\
\frac{\sqrt{4}}{\sqrt{t_{i} t_{j}}}, & \text { for } h_{i j}=\mathbf{i}, \\
\frac{\sqrt{4}}{\sqrt{t_{i} t_{j}}}, & \text { for } h_{i j}=-\mathbf{i}, \\
\frac{1}{\sqrt{t_{i} t_{j}}}, & \text { for } h_{i j}=0 .\end{cases} \\
& \leqslant \frac{2}{\sqrt{t_{i} t_{j}}} \\
& \leqslant \frac{2}{\delta}
\end{aligned}
$$

So, we are going to use $c_{0}=\frac{2}{\delta}$. Next, we consider $\operatorname{Var}\left(Y_{i j}\right)$ for all $1 \leqslant i<j \leqslant n$, and obtain

$$
\operatorname{Var}\left(Y_{i j}\right)=\mathbb{E}\left(\left(Y_{i j}-\mathbb{E}\left(Y_{i j}\right)\right)^{2}\right.
$$




$$
\begin{aligned}
& =\mathbb{E} Y_{i j}^{2} \\
& =\frac{\mathbb{E}\left[\left(h_{i j}-\mathbb{E} h_{i j}\right) H^{i j}+\left(\overline{h_{i j}}-\mathbb{E} \overline{h_{i j}}\right) H^{j i}\right]^{2}}{t_{i} t_{j}} \\
& =\frac{\mathbb{E}\left[\left(h_{i j}-\mathbb{E} h_{i j}\right)\left(\overline{h_{i j}}-\mathbb{E} \overline{h_{i j}}\right)\right]\left(H^{i i}+H^{j j}\right)}{t_{i} t_{j}} \\
& =\frac{\mathbb{E}\left[\left(h_{i j}-\mathbb{E} h_{i j}\right)\left(\overline{h_{i j}-\mathbb{E} h_{i j}}\right)\right]\left(H^{i i}+H^{j j}\right)}{t_{i} t_{j}} \\
& =\frac{\operatorname{Var}\left(h_{i j}\right)\left(H^{i i}+H^{j j}\right)}{t_{i} t_{j}} \\
& =\frac{\left(p_{i j}+p_{j i}+p_{i j} p_{j i}-p_{i j}^{2}-p_{j i}^{2}-p_{i j}^{2} p_{j i}^{2}\right)\left(H^{i i}+H^{j j}\right)}{t_{i} t_{j}} .
\end{aligned}
$$

We also have $\operatorname{Var}\left(Y_{i i}\right)=\mathbb{E} Y_{i i}^{2}=\mathbf{0}$ as $p_{i i}=0$. Therefore,

$$
\begin{aligned}
\left\|\sum_{1 \leqslant i<j \leqslant n} \operatorname{Var}\left(Y_{i j}\right)\right\| & =\left\|\sum_{1 \leqslant i<j \leqslant n} \mathbb{E} Y_{i j}^{2}\right\| \\
& =\left\|\sum_{i=1}^{n} \sum_{j=1}^{n} \frac{\left(p_{i j}+p_{j i}+p_{i j} p_{j i}-p_{i j}^{2}-p_{j i}^{2}-p_{i j}^{2} p_{j i}^{2}\right) H^{i i}}{t_{i} t_{j}}\right\| \\
& =\max _{i=1, \ldots, n} \sum_{j=1}^{n} \frac{p_{i j}+p_{j i}+p_{i j} p_{j i}-p_{i j}^{2}-p_{j i}^{2}-p_{i j}^{2} p_{j i}^{2}}{t_{i} t_{j}} \\
& =\max _{i=1, \ldots, n} \sum_{j=1}^{n} \frac{p_{i j}+p_{j i}-p_{i j} p_{j i}+2 p_{i j} p_{j i}-p_{i j}^{2}-p_{j i}^{2}-p_{i j}^{2} p_{j i}^{2}}{t_{i} t_{j}} \\
& =\max _{i=1, \ldots, n} \sum_{j=1}^{n} \frac{p_{i j}+p_{j i}-p_{i j} p_{j i}-\left(p_{i j}-p_{j i}\right)^{2}-p_{i j}^{2} p_{j i}^{2}}{t_{i} t_{j}} \\
& \leqslant \max _{i=1, \ldots, n} \sum_{j=1}^{n} \frac{p_{i j}+p_{j i}-p_{i j} p_{j i}}{t_{i} t_{j}} \\
& \leqslant \max _{i=1, \ldots, n} \frac{1}{\delta} \sum_{j=1}^{n} \frac{p_{i j}+p_{j i}-p_{i j} p_{j i}}{t_{i}} \\
& =\frac{1}{\delta} .
\end{aligned}
$$

For the final equality, we used that $d_{i}=\sum_{j=1}^{n}\left|h_{i j}\right|$, so $t_{i}=\mathbb{E}\left(d_{i}\right)=\sum_{j=1}^{n} \mathbb{E}\left|h_{i j}\right|=$ $\sum_{j=1}^{n}\left[p_{i j} p_{j i}+p_{i j}\left(1-p_{j i}\right)+\left(1-p_{i j}\right) p_{j i}\right]=\sum_{j=1}^{n}\left(p_{i j}+p_{j i}-p_{i j} p_{j i}\right)$. Now, in order to apply Theorem 8 , we take $a=\sqrt{\frac{4 \ln (4 n / \epsilon)}{\delta}}$, and we let $k$ be large enough so that $\delta>k \ln n$ implies $a<1$ (in particular, choosing $k>4(1+\ln (4 / \epsilon))$ is sufficient). Now, noting that 
$\mathbb{E}\left(\widetilde{\mathcal{L}_{n}}-C_{n}\right)=\mathbf{0}$, applying Theorem 8, we obtain

$$
\begin{aligned}
\operatorname{Pr}\left(\left\|\widetilde{\mathcal{L}_{n}}-C_{n}\right\| \geqslant a\right) & \leqslant 2 n \exp \left(-\frac{a^{2}}{2\left\|\sum_{1 \leqslant i<j \leqslant n} \operatorname{Var}\left(Y_{i j}\right)\right\|+2 c_{0} a / 3}\right) \\
& \leqslant 2 n \exp \left(-\frac{\frac{4 \ln (4 n / \epsilon)}{\delta}}{2 / \delta+4 a / 3 \delta}\right) \\
& =2 n \exp \left(-\frac{4 \ln (4 n / \epsilon)}{2+4 a / 3}\right) \\
& \leqslant 2 n \exp \left(-\frac{4 \ln (4 n / \epsilon)}{4}\right) \\
& =\frac{\epsilon}{2}
\end{aligned}
$$

So, with probability at least $1-\frac{\epsilon}{2},\left\|\widetilde{\mathcal{L}_{n}}-C_{n}\right\| \leqslant a$. For the second term, we first rewrite $C_{n}-\mathcal{L}_{n}$, as follows.

$$
\begin{aligned}
C_{n}-\mathcal{L}_{n}= & I_{n}-\left(\mathbb{E} D_{n}\right)^{-1 / 2} H_{n}\left(\mathbb{E} D_{n}\right)^{-1 / 2}-I_{n}+D_{n}^{-1 / 2} H_{n} D_{n}^{-1 / 2} \\
= & D_{n}^{-1 / 2} H_{n} D_{n}^{-1 / 2}-\left(\mathbb{E} D_{n}\right)^{-1 / 2} D_{n}^{1 / 2} D_{n}^{-1 / 2} H_{n} D_{n}^{-1 / 2} D_{n}^{1 / 2}\left(\mathbb{E} D_{n}\right)^{-1 / 2} \\
= & I_{n}-\mathcal{L}_{n}-\left(\mathbb{E} D_{n}\right)^{-1 / 2} D_{n}^{1 / 2}\left(I_{n}-\mathcal{L}_{n}\right) D_{n}^{1 / 2}\left(\mathbb{E} D_{n}\right)^{-1 / 2} \\
= & \left(I_{n}-\mathcal{L}_{n}\right)-\left(I_{n}-\mathcal{L}_{n}\right) D_{n}^{1 / 2}\left(\mathbb{E} D_{n}\right)^{-1 / 2} \\
& -\left(\mathbb{E} D_{n}\right)^{-1 / 2} D_{n}^{1 / 2}\left(I_{n}-\mathcal{L}_{n}\right) D_{n}^{1 / 2}\left(\mathbb{E} D_{n}\right)^{-1 / 2}+\left(I_{n}-\mathcal{L}_{n}\right) D_{n}^{1 / 2}\left(\mathbb{E} D_{n}\right)^{-1 / 2} \\
= & \left(I_{n}-\mathcal{L}_{n}\right)\left[I_{n}-D_{n}^{1 / 2}\left(\mathbb{E} D_{n}\right)^{-1 / 2}\right] \\
& +\left[I_{n}-\left(\mathbb{E} D_{n}\right)^{-1 / 2} D_{n}^{1 / 2}\right]\left(I_{n}-\mathcal{L}_{n}\right) D_{n}^{1 / 2}\left(\mathbb{E} D_{n}\right)^{-1 / 2}
\end{aligned}
$$

Recalling that $\left\|I_{n}-\mathcal{L}_{n}\right\| \leqslant 1$, we obtain the following expression for $\left\|C_{n}-\mathcal{L}_{n}\right\|$.

$$
\begin{aligned}
\left\|C_{n}-\mathcal{L}_{n}\right\| \leqslant & \left\|I_{n}-\mathcal{L}_{n}\right\|\left\|I_{n}-D_{n}^{1 / 2}\left(\mathbb{E} D_{n}\right)^{-1 / 2}\right\| \\
& +\left\|I_{n}-\left(\mathbb{E} D_{n}\right)^{-1 / 2} D_{n}^{1 / 2}\right\|\left\|I_{n}-\mathcal{L}_{n}\right\|\left\|D_{n}^{1 / 2}\left(\mathbb{E} D_{n}\right)^{-1 / 2}\right\| \\
\leqslant & \left\|I_{n}-D_{n}^{1 / 2}\left(\mathbb{E} D_{n}\right)^{-1 / 2}\right\|+\left\|I_{n}-\left(\mathbb{E} D_{n}\right)^{-1 / 2} D_{n}^{1 / 2}\right\|\left\|D_{n}^{1 / 2}\left(\mathbb{E} D_{n}\right)^{-1 / 2}\right\| .
\end{aligned}
$$

Next, we are going to obtain an upper bound for $\left\|I_{n}-D_{n}^{1 / 2}\left(\mathbb{E} D_{n}\right)^{-1 / 2}\right\|$. For this, we will apply Lemma 13 to the random variables $\left|h_{i j}\right|$ (in the role of $X_{i}$ ), and using the observations that $d_{i}=\sum_{j=1}^{n}\left|h_{i j}\right|$, and $t_{i}=\mathbb{E}\left(d_{i}\right)=\sum_{j=1}^{n} \mathbb{E}\left|h_{i j}\right|$. We first need some preparation in order to obtain upper bounds for $\left|h_{i j}\right|$ and $\sum_{j=1}^{n} \operatorname{Var}\left(\left|h_{i j}\right|\right)$.

Obviously, $\left|h_{i j}\right| \leqslant 1$, so we can take $c=1$, and

$$
\sum_{j=1}^{n} \operatorname{Var}\left(\left|h_{i j}\right|\right)=\sum_{j=1}^{n}\left[\mathbb{E}\left(\left|h_{i j}\right|^{2}\right)-\left(\mathbb{E}\left(\left|h_{i j}\right|\right)\right)^{2}\right]
$$




$$
\begin{aligned}
& =\sum_{j=1}^{n}\left[p_{i j}+p_{j i}-p_{i j} p_{j i}-\left(p_{i j}+p_{j i}-p_{i j} p_{j i}\right)^{2}\right] \\
& \leqslant \sum_{j=1}^{n}\left(p_{i j}+p_{j i}-p_{i j} p_{j i}\right) \\
& =\sum_{j=1}^{n} \mathbb{E}\left|h_{i j}\right| \\
& =t_{i} .
\end{aligned}
$$

Since we already used $a$ above, with $a=\sqrt{\frac{4 \ln (4 n / \epsilon)}{\delta}}<1$, we are going to use a $b$ instead of an $a$ when applying Lemma 13. We choose $b=\sqrt{3 t_{i} \ln (4 n / \epsilon)}$. Then, since $a=$ $\sqrt{\frac{4 \ln (4 n / \epsilon)}{\delta}}<1, t_{i} \geqslant \delta>4 \ln (4 n / \epsilon)$, implying that $b=\sqrt{3 t_{i} \ln (4 n / \epsilon)}<t_{i}$. Applying Lemma 13, we have for all $i$,

$$
\operatorname{Pr}\left(\left|d_{i}-t_{i}\right| \geqslant b\right) \leqslant e^{-\frac{b^{2}}{2\left(t_{i}+b / 3\right)}}<e^{-\frac{3 t_{i} \ln (4 n / \epsilon)}{3 t_{i}}}=\frac{\epsilon}{4 n} .
$$

This implies that with probability at least $1-o(1)$, for all $1 \leqslant i \leqslant n,\left|d_{i}-t_{i}\right| \leqslant b=$ $\sqrt{3 t_{i} \ln (4 n / \epsilon)}$.

Next, we use the known fact that $|\sqrt{x}-1| \leqslant|x-1|$ for any real number $x>0$. Taking $x=\frac{d_{i}}{t_{i}}>0$, we obtain that with probability at least $1-\frac{\epsilon}{2}$,

$$
\left|\sqrt{\frac{d_{i}}{t_{i}}}-1\right| \leqslant\left|\frac{d_{i}}{t_{i}}-1\right|=\frac{\left|d_{i}-t_{i}\right|}{t_{i}} \leqslant \frac{\sqrt{3 t_{i} \ln (4 n / \epsilon)}}{t_{i}} \leqslant \sqrt{\frac{3 \ln (4 n / \epsilon)}{\delta}}=\frac{\sqrt{3}}{2} a .
$$

Thus, we obtain

$$
\left\|I_{n}-D_{n}^{1 / 2}\left(\mathbb{E} D_{n}\right)^{-1 / 2}\right\|=\max _{i=1,2, \ldots, n}\left|\sqrt{\frac{d_{i}}{t_{i}}}-1\right| \leqslant \frac{\sqrt{3}}{2} a
$$

with probability at least $1-\frac{\epsilon}{2}$. So, with probability at least $1-\frac{\epsilon}{2}$,

$$
\begin{aligned}
\left\|C_{n}-\mathcal{L}_{n}\right\| & \leqslant\left\|I_{n}-D_{n}^{1 / 2}\left(\mathbb{E} D_{n}\right)^{-1 / 2}\right\|+\left\|I_{n}-\left(\mathbb{E} D_{n}\right)^{-1 / 2} D_{n}^{1 / 2}\right\|\left\|D_{n}^{1 / 2}\left(\mathbb{E} D_{n}\right)^{-1 / 2}\right\| \\
& \leqslant \frac{\sqrt{3}}{2} a+\frac{\sqrt{3}}{2} a\left(\frac{\sqrt{3}}{2} a+1\right) \\
& =\frac{3}{4} a^{2}+\sqrt{3} a .
\end{aligned}
$$

Combining the above bound with the bound we obtained for $\left\|C_{n}-\widetilde{\mathcal{L}_{n}}\right\|$, and using that $a<1$, we conclude that with probability at least $1-\epsilon$,

$$
\begin{aligned}
\left\|\mathcal{L}_{n}-\widetilde{\mathcal{L}_{n}}\right\| & \leqslant\left\|C_{n}-\widetilde{\mathcal{L}_{n}}\right\|+\left\|C_{n}-\mathcal{L}_{n}\right\| \\
& \leqslant a+\frac{3}{4} a^{2}+\sqrt{3} a
\end{aligned}
$$




$$
\begin{aligned}
& \leqslant \frac{7}{2} a \\
& =\frac{7}{2} \sqrt{\frac{4 \ln (4 n / \epsilon)}{\delta}} \\
& =7 \sqrt{\frac{\ln (4 n / \epsilon)}{\delta}} .
\end{aligned}
$$

For the final step in our proof, we use Lemma 12, which states that for Hermitian matrices $M$ and $N, \max _{k}\left|\lambda_{k}(M)-\lambda_{k}(N)\right| \leqslant\|M-N\|$. Thus, with probability at least $1-\epsilon$, we have that for all $1 \leqslant i \leqslant n$,

$$
\left|\lambda_{i}\left(\mathcal{L}_{n}\right)-\lambda_{i}\left(\widetilde{\mathcal{L}_{n}}\right)\right| \leqslant\left\|\mathcal{L}_{n}-\widetilde{\mathcal{L}_{n}}\right\| \leqslant 7 \sqrt{\frac{\ln (4 n / \epsilon)}{\delta}}
$$

This completes the proof of Theorem 4 .

\section{Acknowledgements}

We thank the anonymous referees for their helpful comments and suggestions.

\section{References}

[1] R. Ahlswede and A. Winter. Strong converse for identification via quantum channels. IEEE Trans. Inform. Theory, 48(3) (2002), 569-579.

[2] N. Alon, M. Krivelevich, and V. H. Vu. Concentration of eigenvalues of random matrices. Israel Math. J., 131 (2002), 259-267.

[3] R. Bhatia. Matrix Analysis, Graduate Texts in Mathematics, vol. 169, Springer, Berlin, 1997, p.10.

[4] R. Bhatia. Positive Definite Matrices, Princeton Univ. Press, Princeton, 2007.

[5] F. Chung. Spectral graph theory, AMS publications, 1997.

[6] F. Chung, L. Lu, and V. H. Vu. Eigenvalues of random power law graphs. Ann. Combin., 7 (2003), 21-33.

[7] F. Chung, L. Lu, and V. H. Vu. Spectra of random graphs with given expected degrees. Proc. Nat. Acad. Sci. USA, 100(11) (2003), 6313-6318.

[8] F. Chung and M. Radcliffe. On the spectra of general random graphs. Electron. J. Combin., 18 (2011), \#P215.

[9] A. Coja-Oghlan. On the Laplacian eigenvalues of $G(n, p)$. Combin. Probab. Comput., 16 (2007), 923-946.

[10] A. Coja-Oghlan and A. Lanka. The spectral gap of random graphs with given expected degrees. Electron. J. Combin., 16 (2009), \#R138.

[11] D. Cristofides and K. Markström. Expansion properties of random Cayley graphs and vertex transitive graphs via matrix martingales. Random Struct. Alg., 32 (2008), $88-100$. 
[12] D. M. Cvetković, M. Doob, and H. Sachs. Spectra of Graphs, Theory and Applications, Academic Press, 1980.

[13] X. Ding and T. Jiang. Spectral distributions of adjacency and Laplacian matrices of random graphs. Ann. Appl. Probab., 20 (2010), 2086-2117.

[14] U. Feige and E. Ofek. Spectral techniques applied to sparse random graphs. Random Struct. Alg., 27(2) (2005), 251-275.

[15] J. Friedman. A Proof of Alon's Second Eigenvalue Conjecture and Related Problem, Memoirs of the American Mathematical Society 2008, 100 pp.

[16] J. Friedman. On the second eigenvalue and random walks in random $d$-regular graphs. Combinatorica, 11(4) (1991), 331-362.

[17] J. Friedman, J. Kahn, and E. Szemerédi. On the second eigenvalue in random regular graphs, in Proc. 21st ACM Symp. Theory of Computing, 1989, 587-598.

[18] Z. Füredi and J. Komlós. The eigenvalues of random symmetric matrices. Combinatorica, 1(3) 1981, 233-241.

[19] D. Gross. Recovering low-rank matrices from few coefficients in any basis. IEEE Trans. Inform. Theory, 57 (2011), 1548-1566.

[20] K. Guo and B. Mohar. Hermitian adjacency matrix of digraphs and mixed graphs. J. Graph Theory, 85 (2017), no. 1, 217-248.

[21] R. A. Horn and C. R. Johnson. Matrix Analysis, 2nd, Cambridge University Press, 2012.

[22] M. Krivelevich and B. Sudakov. The largest eigenvalue of sparse random graphs. Combin. Probab. Comput., 12 (2003), 61-72.

[23] J. Liu and X. Li. Hermitian-adjacency matrices and Hermitian energies of mixed graphs. Linear. Algebra. Appl., 466 (2015) 182-207.

[24] L. Lu and X. Peng. Loose Laplacian spectra of random hypergraphs. Random Struct. Alg., 41 (2012), no. 4, 521-545.

[25] L. Lu and X. Peng. Spectra of edge-independent random graphs. Electron. J. Combin., 20(4) (2013), \#P27.

[26] R. Oliveira. Concentration of the adjacency matrix and of the Laplacian in random graphs with independent edges. arXiv:0911.0600, 2009.

[27] D. Petz. A survey of certain trace inequalities, in Functional Analysis and Operator Theory, Banach Center Publications, vol. 30(Polish Acad. Sci., Warsaw, 1994), pp. 287-298.

[28] B. Recht. Simpler approach to matrix completion. J. Mach. Learn. Res., 12 (2011), 3413-3430.

[29] J. Tropp. User-friendly tail bounds for sums of random matrices. Found. Comput. Math., 12 (2012), 389-434.

[30] H. Weyl. Das asymptotische Verteilungsgesetz der Eigenwerte linearer partieller Differentialgleichungen. Math. Ann., 71 (2010), 441-479.

[31] F. Zhang. Matrix theory, Springer-Verlag, New York, 1999. 